\title{
Software Estadístico CHIC: descubriendo sus potencialidades mediante el análisis de percepción sexual universitaria.
}

\section{Statistical Software Chic: discovering their potential through the analysis of sexual perception university education.}

Rubén Pazmiño. ${ }^{1}$, Marina Bonilla. ${ }^{2}$, Jenner Baquero. ${ }^{3}$ \& Rogel Miguez. ${ }^{4}$

Recibido: 06-07-2017 / Revisado: 16-09-2018 Aceptado: 15-10-2018/ Publicado: 01-11-2018

\begin{abstract}
.
DOI: https://doi.org/10.33262/cienciadigital.v2i4.1..194

Statistical Implicative Analysis is a multivariate statistic non-parametric technique to knowledge discovering in databases using asymmetric R-rules within variables and variables classes. Its has been discovered by the French Regis Gras, 40 years ago and is constantly growing in theoretical, computer and application. The computer tool which automates the Statistical Implicative Analysis is called CHIC. In 2015, the Escuela Superior Politécnica de Chimborazo has finished to development the free version called R-CHIC, its development was made by Raphael Couturier. The methodology used was knowledge discover in databases with the Statistical Implicative Analysis applied to 198 variables and 100 subjects. This communication provides installation, execution, R-CHIC application and its interpretation in sexual perception context, for first time. The main results were the steps to install and execute R-CHIC, the Statistical Implicative Analysis application using similarity trees, hierarchical trees, implicative graphs and its interpretation. Concludes with interesting interpretations of student sexual perception, based on R-CHIC software using symmetrical and asymmetric relationship rules, in a fast, intuitive, visual and comprehensible manner.

\footnotetext{
${ }^{1}$ Escuela Superior Politécnica de Chimborazo, Facultad de Ciencias, Grupo de investigación Ciencia de Datos (CIDED), Riobamba, Ecuador, rpazmino@espoch.edu.ec

${ }^{2}$ Escuela Superior Politécnica de Chimborazo, Facultad de Ciencias, Grupo de investigación Ciencia de Datos (CIDED), Riobamba, Ecuador, marina.bonilla@espoch.edu.ec

${ }^{3}$ Escuela Superior Politécnica de Chimborazo, Facultad de Ciencias, Grupo de investigación Ciencia de Datos (CIDED), Riobamba, Ecuador, jenner.baquero@espoch.edu.ec

${ }^{4}$ Escuela Superior Politécnica de Chimborazo, Facultad de Ciencias, Grupo de investigación Ciencia de Datos (CIDED), Riobamba, Ecuador, rogel.miguez@espoch.edu.ec
} 
Keywords: R-CHIC, Statistical Implicative Analysis, sexual perception, ESPOCH.

\section{Resumen.}

El Análisis Estadístico Implicativo es una técnica estadística no paramétrica multivariada para descubrir conocimiento en Bases de Datos mediante R-reglas asimétricas entre variables y clases de variables. Fue descubierta por el francés Regis Gras aproximadamente hace 40 años y está en constante crecimiento en su parte teórica, computacional y aplicaciones. La herramienta computacional que automatiza el Análisis Estadístico Implicativo es llamada CHIC, en el año 2015 se finalizó en la Escuela Superior Politécnica de Chimborazo el desarrollo de su versión libre llamada R-CHIC, su desarrollo estuvo a cargo del francés Raphael Couturier. La metodología utilizada fue la del descubrimiento de conocimiento en bases de datos con el Análisis Estadístico Implicativo aplicado a una base de datos de 198 variables y 100 sujetos. Esta comunicación aporta mostrando la instalación, ejecución y además la aplicación del software estadístico R-CHIC y su interpretación en el contexto de la percepción sexual, por primera ocasión. Como principales resultados se obtuvieron los pasos para la instalación y ejecución de R-CHIC, la aplicación del Análisis Estadístico Implicativo mediante árboles de similaridad, árboles jerárquicos y grafos Implicativos y su interpretación. Se concluye con interpretaciones interesantes a la percepción sexual estudiantil basadas en el software R-CHIC mediante reglas de relación simétricas y asimétricas en forma rápida, visual, intuitiva y comprensible.

Palabras Claves: R-CHIC, Análisis Estadístico Implicativo, percepción sexual, Escuela Superior Politécnica de Chimborazo.

\section{Introducción.}

Esta sección permite un primer acercamiento al Análisis Estadístico Implicativo, el software CHIC y realiza una aproximación a la percepción sexual en general y a la percepción sexual en la Escuela Superior Politécnica en particular.

\section{El Análisis Estadístico Implicativo.}

El análisis estadístico Implicativo (ASI, del francés Analisi Statistica Implicativa), es una teoría estadística desarrollada por el francés Regis Gras en los años 80, utiliza el concepto de cuasi-implicación para generar relaciones asimétricas entre variables basadas en r-reglas (Couturier \& Pazmiño, 2016). La cuasi-implicación p->q se produce cuando la variable p es observada entonces generalmente la variable q también lo es, es decir es una implicación lógica, pero con pocas excepciones. A diferencia de los métodos de análisis simétricos (Regnier, 2009) los conjuntos de reglas obtenidos pueden conducir a hipótesis de causalidad (GRAS \& COUTURIER, 2012). Una de las representaciones gráficas que utiliza el análisis 
estadístico Implicativo es el dendograma que es un tipo de diagrama de árbol utilizado en las agrupaciones jerárquicas, para representar relaciones entre variables en diferentes niveles de similaridad, los nodos significativos son nodos correspondientes a una clasificación compatible lo mejor posible con los valores y la calidad del agrupamiento obtenido. .Desde sus orígenes la teoría del ASI ha sido aplicada en la generación y gestión de conocimiento en educación, psicología, matemática, arte, medicina pero no en el estudio de la sexualidad (Pazmiño, 2014), llevándose a formalizar su relación con el descubrimiento de conocimiento en bases de datos (KDD) en el artículo (Pazmiño-Maji, García-Peñalvo, \& Conde-González, 2017). CHIC (del francés Classification Hiérarchique, Implicative \& Cohésitive) y R-CHIC (CHIC en el ambiente estadístico R) son programas desarrollado por Raphaël Couturier que automatiza la teoría del análisis estadístico Implicativo (Couturier, 2007) (Pazmiño-Maji, García-Peñalvo, \& Conde-González, 2016).

\section{El Software Estadístico CHIC.}

La génesis histórica y la evolución del software CHIC se da en paralelo con los desarrollos teóricos del ASI. R. Gras, en 1979 construye el índice de implicación estadística entre variables binarias, más tarde junto con su equipo amplían el estudio de la implicación al considerar en cada etapa de nuevos problemas planteados las nociones matemáticas y analizar otro tipo de relación entre variables: no binarias, de intervalo, de intervalo y difusas, de cualquier tipo (Régnier \& Gras, 2015). Existe la necesidad de nuevos desarrollos teóricos que consideren el concepto de "inclusión de implicaciones", nociones de tipicalidad y la contribución de variables adicionales, nodos significativos, apoyándose en la herramienta informática CHIC (Ribeiro, Santos, Vilardell, Carolei, \& Mengali, 2015). Antes de 1990, el software CHIC fue una versión primitiva realizada por Regis Gras y programada en Basic para microcomputadora en 1984, donde se implementó principalmente el cálculo de las intensidades de implicación (Gras, 2014). En los años 1990-1992, S. Almouloud, realizó una herramienta informática confiable y amigable, un software de análisis de datos para permitir la integración de varios tratamientos, además del análisis de similitud de IC Lerman, el análisis implicativo de Regis Gras y sus extensiones como jerarquía implicativa de clase y estudio de variables numéricas y modales. Esta primera edición de datos conlleva a restricciones como la imposibilidad de agregar o eliminar variables o individuos, entre otras, siendo mejorado progresivamente por S. Ag Almouloud aumentando la extensión de la tabla de datos, la concatenación de dos archivos con el mismo número de individuos, modificación de valor de un dato, reproducción del archivo de datos, mejor gestión de memoria y puertos gráficos, el cálculo de las intensidades de implicación (Couturier \& Almouloud, 2009). El principal cambio que hizo luego es la precisión de los resultados y con A. Totohasina se simula la ley normal. La última versión de CHIC realizada por z S. Ag Almouloud fue escrita en turbo pascal 6, consistía en un programa principal y un conjunto de subprogramas: la edición, clasificación de similitud jerárquica, nodos significativos, implicación de variables, cálculo de parámetros, construcción del grado implicativo y jerarquía implicactiva y nodos 
significativos (Couturier \& Almouloud, 2009). En paralelo Harrisson Ratsimba Rajohn introdujo la noción de tipicalidad de variables adicionales e individuos y realizó el programa informático que permitia su tratamiento. Desde 1993, Raphaël Couturier ha proporcionado mejoras y una evolución del software CHIC considerando los nuevos desarrollos teóricos de ASI y los deseos de los usuarios; dando lugar a docenas de versiones diferentes hasta la versión actual 7.0 realizada en $\mathrm{C}$, que permite tratar diferentes variables, que no sean binarias, cuantificar la importancia de los valores atribuidos a la calidad, la consistencia de la regla asociadas, de las clases de reglas ordenadas, la tipicalidad y la contribución de sujetos o categorías de sujetos a ciertas reglas, representar un umbral de calidad elegido, rutas de reglas y reglas generalizadas y eliminar, agregar y asociar variables (COUTURIER, 2000). Actualmente el software CHIC procesa variables binarias (caso más simple), variables de frecuencia (valor entre 0 y 1 ), variables definidas por intervalo y variables de intervalo (se usan para modelar situaciones complejas). En el año 2015 se finalizó en la Escuela Superior Politécnica de Chimborazo el desarrollo de la versión libre de CHIC, llamada R-CHIC, por el francés Raphael Couturier. Tanto CHIC como R-CHIC utilizan el formato CSV como el formato de datos para los archivos de datos, se pueden usar variables adicionales para la formación de ciertas reglas, se usa en el cálculo de tipicalidad y contribución. CHIC a diferencia de R-CHIC, ofrece otras herramientas interesantes como el cálculo de la contribución y la tipicalidad de un individuo o un conjunto de individuos a una regla dada, en cada representación gráfica.

\section{La percepción sexual.}

La percepción es el proceso en el cual registramos todo lo que está a nuestro alrededor o en interacción con nosotros, en forma tal que tiene un significado. Incluye los valores, las tradiciones, los estereotipos, las vivencias y los conocimientos que tienen las personas sobre determinados aspectos o situaciones de la vida (Calero \& Santana, 2001). El entorno, el medio de socialización y las personas con las cuales se convive durante la adolescencia juega un papel importante en la educación, en la formación y transmisión de valores, los que definen sus percepciones y comportamientos relacionado a su sexualidad. El término sexualidad aparentemente desconocido, pero que no siempre somos capaces de definir; la palabra sexualidad se incorpora al vocabulario envuelta en mitos, temores, signos de interrogación y expectativas. Los investigadores desde sus disciplinas científicas y posiciones filosóficas (médicos, psicólogos, pedagogos, biólogos), han propuesto conceptos que intentan definir la sexualidad humana (Ciencias, Granma, Universitario, Hirzel, \& Granma, 2016). La sexualidad es concebida como el conjunto de características biológicas, psicológicas y socioculturales, acompañadas de creencias, comportamientos, sentimientos, actitudes, pensamientos y valores presentes en toda la vida del individuo permitiéndole expresarse y relacionarse (Hurtado, Zabalgoitia, \& Olvera, 2013). La sexualidad es considerada como una construcción social, en la que se involucran nuestras emociones, deseos y relaciones, expresadas en la sociedad en que vivimos (Adela Montero, 2011), es una 
parte de la personalidad e identidad y una de las necesidades humanas que se expresa a través del cuerpo, el elemento básico de la femineidad y masculinidad, de la autoimagen, la autoconciencia (Ciencias et al., 2016). No se puede comprender la sexualidad humana sin reconocer de antemano su índole pluridimensional (Guerra Electra, 2016). La sexualidad de cada uno de nosotros emerge y existe en un contexto cultural, influenciada por variables tales como psicológicas, biológicas, socio económicas, edad, rol de género, etnicidad, siendo imprescindible el reconocimiento y respeto de esta variabilidad de formas, creencias y conductas sexuales (Adela Montero, 2011). El conocimiento de la sexualidad contribuye a que las personas sean más plenas y afronten la vida sexual con cierta preparación, el conocimiento sobre sexualidad en la juventud es esencial para lograr una sexualidad responsable (Guerra Electra, 2016). Según la definición de las Naciones Unidas, los jóvenes son las personas con edades comprendidas entre los 15 y los 24 años. La UNESCO entiende que los jóvenes constituyen un grupo heterogéneo en constante evolución y que la experiencia de "ser joven", varía mucho de acuerdo con las regiones del planeta e incluso de un mismo país. La categoría juventud ha sido concebida como una construcción social, histórica, cultural y relacional. Una etapa de vida que tiene sus propias oportunidades y limitaciones, en la cual lo más característico es la construcción de identidad a un nivel personal, generacional y social que se asocian a condiciones individuales, familiares, sociales, culturales e históricas determinadas (Dávila León, 2004). El conocimiento sobre sexualidad en la juventud es esencial para lograr una sexualidad responsable (Guerra Electra, 2016), la adolescencia y juventud son edades tempranas favorables para modificar enseñanzas rígidas, mitos, tabúes, creencias y formas de conductas que hayan influenciado en su formación, permitiéndoles manifestar autorresponsabilidad en el cuidado de la salud en la etapa de adultos (Cabrera, Ramos, Palú, \& Cáceres, 2013). Entre los factores que incrementan la vulnerabilidad de los jóvenes destacan el consumo de sustancias como el alcohol y otras drogas, dificultades de negociación de preservativo y el uso de internet para contactar parejas sexuales (Folch, Álvarez, Casabona, Brotons, \& Castellsagué, 2015). Según un estudio realizado el consumir bebidas alcohólicas en la adolescencia se vincula con al menos tres indicadores de riesgo sexual como frecuencia de relaciones sexuales, menor uso del condón y mayor número de parejas sexuales, la asociación entre los indicadores del consumo de alcohol y la conducta sexual señala que, consumir bebidas alcohólicas por primera vez concuerda con la edad de la primera relación sexual (Delgado, Flores, Palos, Adolescentes, \& Consumo De Alcohol, 2007). Según la OMS (2014) se reconoce más la importancia de abordar la salud sexual y reproductiva de los adolescentes. En la asamblea Mundial de la Salud de la OMS 2018 destacan cómo las desigualdades de género y la discriminación siguen alimentando un número desproporcionado de nuevas infecciones de VIH en mujeres y adolescentes, garantizar el acceso de niñas y mujeres a la salud es clave para lograr la visión de 2018. Según estudios realizados es necesario continuar promoviendo el uso de métodos anticonceptivos y preventivos entre los y las jóvenes para consumar relaciones sexuales protegidas y de esta manera poder disfrutar de una sexualidad saludable 
y placentera. El diseño de una estrategia educativa conduce a fomentar un pensamiento responsable sobre salud sexual y reproductiva en los jóvenes (Cabrera et al., 2013).

\section{La percepción sexual en la ESPOCH.}

La Escuela Superior Politécnica de Chimborazo (ESPOCH), tiene su origen en el Instituto tecnológico Superior de Chimborazo, creado mediante Ley No.69,09, expedida por el Congreso Nacional, el 18 de abril de 1969, inicia sus actividades académicas el 2 de mayo de 1972 ("Inicio - Escuela Superior Politécnica de Chimborazo,"). Existen pocos estudios previos sobre percepción sexual en la Escuela Superior Politécnica de Chimborazo a pesar de que se han registrado algunos esfuerzos académicos que han ido desde algún congreso internacional sobre sexualidad, hasta la implementación de un sistema contra el acoso sexual; el estudiante sin embargo, continúa siendo un elemento pasivo de estudio. Según un estudio realizado en la ESPOCH se constata que la educación sexual es deficiente o nula entre los jóvenes universitarios y que la familia, la escuela y los diferentes espacios de socialización, no figuran como ejes formativos en este ámbito. Romper el silencio sobre las relaciones sexuales, la anticoncepción y las Infecciones de Transmisión Sexual es fundamental para acabar con los mitos y permitir a los jóvenes tomar decisiones informadas en relación con sus necesidades individuales (Hernández, del Carmen, Pérez Piñero, \& Sanabria Ramos, 2013).

Para tener información sobre la percepción sexual en la ESPOCH, se pidió a cinco grupos de 5 encuestadores que durante una semana desde las 7:00 hasta las 21:00 horas se aplique aleatoriamente una entrevista no estructurada; cada equipo entrevistó a 20 estudiantes de diferentes carreras y edades de la ESPOCH, acerca de sus percepciones vinculadas a las prácticas, costumbres y criterios de su realidad sexual esto se lo hizo sin inducir y exponer indicadores o variables idiosincráticas inductivas para no incurrir en sesgos culturales.

\section{Materiales y métodos.}

Los materiales utilizados fueron la Base de datos de Percepción Sexual y el software estadístico libre R-CHIC, a continuación describimos detalladamente cada uno de ellos.

\section{La Base de datos de Percepción Sexual y el software estadístico libre R-CHIC.}

Se analizaron las entrevistas, extrayéndose de estas, las palabras clave de cada criterio, estas vinieron a constituir las comunalidades del estudio, cuya frecuencia analizada por triangulación arrojó el verificador de comunidad de criterios. Se realizó un estudio multivariado de correspondencias de los datos de las variables con fines de validación factorial de los datos, esto, posterior a la respectiva validación de fiabilidad. Finalmente se perfiló un estudio de la percepción sexual de los estudiantes mediante el software R-CHIC. A continuación se muestran en detalle los pasos para la instalación y ejecución del software $\mathrm{R}-\mathrm{CHIC}$ 
1) Instalar $R$ de la página web

2) Instalar de la página web RStudio (Pazmiño, 2014)

3) Crear un proyecto en RStudio,

4) Crear un R-script en RStudio

5) Instalar y activar el paquete Rgraphviz de la página web ("Bioconductor - Rgraphviz,")

6) Instalar y activar los paquetes stringr, tcltk2 y Rcpp.

7) Acceder a la página oficial de R-CHIC ("Rchic - | Raphael Couturier,")

8) Descargar, instalar y activar el paquete R-CHIC

9) Preparar los datos en formato csv (del inglés a comma separated values)

10) Ubicar la base en formato csv en la carpeta del proyecto

11) Para la ejecución activar el menú de R-CHIC utilizando el comando rchic()

12) Seleccionar una de las tres opciones de cálculo: Complete graph, Classic, Classic + confidence, Implifiance, ver Figura 1.a.

13) Seleccionar una de las opciones del menu: Similarity tree, Hierarchi tree, Implicative graph (ver Figura 1.b.); y finalmente

14) Descubrir el conocimiento en los resultados obtenidos:
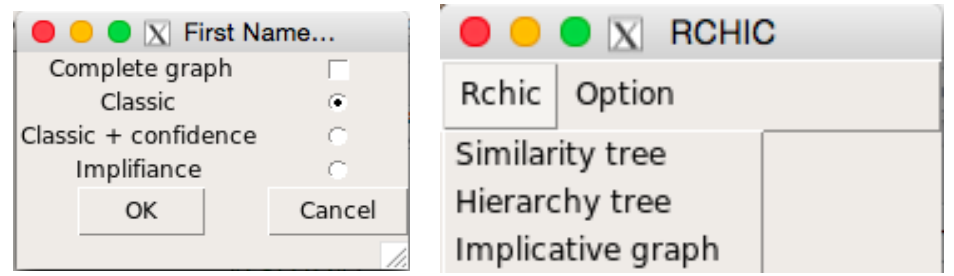

Figura 1.a y 1.b. Opciones de cálculo y del menú del R-CHIC

\section{El Método del Análisis Estadístico Implicativo.}

La metodología utilizada se ha tomado de (Pazmiño-Maji et al., 2017) y se basa en los pasos que constituyen el proceso del Análisis Estadístico Implicativo desde el punto de vista del descubrimiento de conocimiento en bases de datos KDD (del inglés Knowledge DataBase). A continuación, se detallan los pasos aplicados en la metodología (Ver Figura 2.):

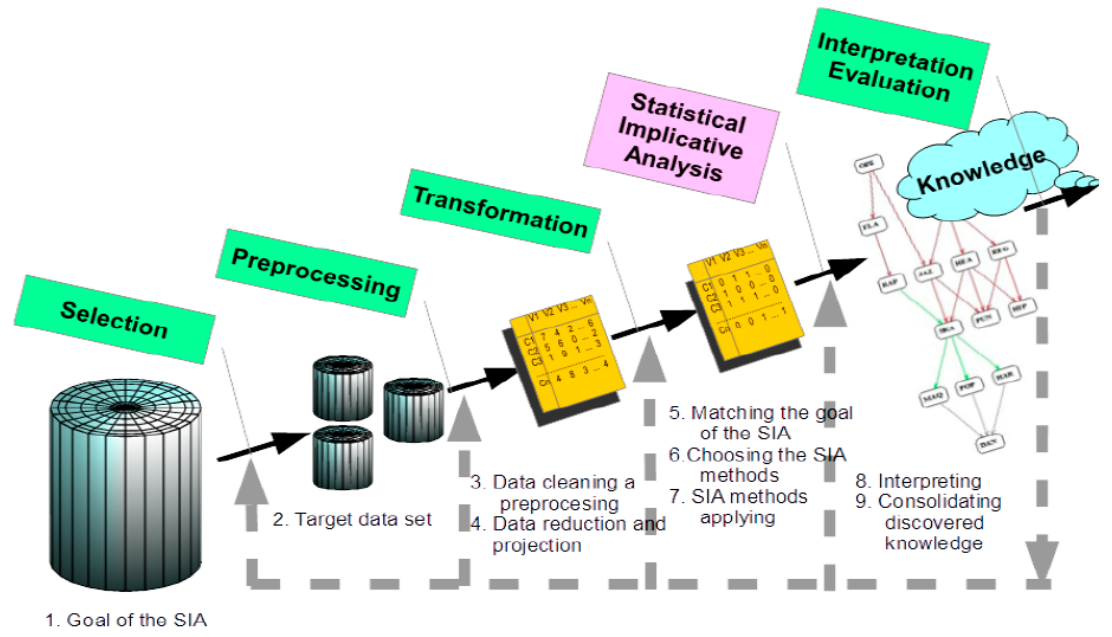

Figura 2. Metodología basada en el descubrimiento de conocimiento en bases de 
datos KDD

Selección: Se realizó una selección aleatoria de 100 estudiantes universitarios de diferentes carreras y edades de la ESPOCH y se les aplicó una entrevista no estructurada; acerca de sus percepciones sexuales. La muestra fue tomada considerando futuros estudios inferenciales, que la principal fuente de datos son los estudiantes de la Facultad de Ciencias, que según el informe del Análisis Socio-Económico de la Población de Estudiantes de la ESPOCH la Facultad de Ciencias tiene un promedio aproximado de 948 estudiantes y aplicando la fórmula de muestreo de proporciones con población conocida, un valor alfa de 0.1 y un error máximo permitido de 0.08 se obtuvo un tamaño muestral mayor o igual a 95.175 y que lo aproximamos con 100.

Preprocesamiento: La entrevista realizada se digitalizó en una hoja de cálculo en excel obteniéndose una base de datos estadística de 100 filas por 60 columnas.

Transformación: La base de datos obtenida se transformó en una base de datos dicotómica en formato de texto separado por comas (*.csv) conformado por una total de 100 filas correspondientes a los trabajos de titulación y 649 columnas, correspondientes a la información codificada.

Análisis estadístico Implicativo: Se lo aplicó utilizando el software R-CHIC versión 0.25, con él se obtuvieron árboles de similaridad, grafos implicativos y árboles de jerarquicos que se basaron en los datos en formato plano separado por comas y luego se exportaron en formato JPEG.

Interpretación y Evaluación: La generación, interpretación y evaluación del nuevo conocimiento se basaron en los gráficos obtenidos que muestran mediante los grafos de implicación las R-Reglas, los árboles de similaridad que muestra las variables similares utilizando el criterio de similaridad de Israel Lerman y el árbol jerárquico que permite ver mediante un dendograma las diferentes relaciones asimétricas entre variables y clase de variables.

\section{Resultados y discusión.}

Para los ejemplos de aplicación a la base de datos de percepción sexual y su discusión se utilizaron variables extraidas de la entrevista no estructurada, que se muestran a continuación en la Tabla 1: 
Tabla 1.Algunas preguntas y sus posibles respuestas de la entrevista no estructurada sobre percepción sexual.

\begin{tabular}{|c|c|}
\hline Preguntas & Respuestas \\
\hline $\begin{array}{l}\text { ¿Cuál es la primera } \\
\text { palabra que asocias con } \\
\text { sexo? }\end{array}$ & $\begin{array}{l}\text { v051.P11.amor, v051.P11.caricias, v051.P11.cariño, v051.P11.comprensión, } \\
\text { v051.P11.deseo, v051.P11.felicidad, v051.P11.hombre, v051.P11.intimidad, } \\
\text { v051.P11.mujer, v051.P11.pareja, v051.P11.pasión, v051.P11.placer, } \\
\text { v051.P11.satisfacción, v051.P11.vida }\end{array}$ \\
\hline $\begin{array}{l}\text { ¿Cuáles son los motivos } \\
\text { que te impulsaron a } \\
\text { tener tu primera } \\
\text { relación? }\end{array}$ & $\begin{array}{l}\text { v053.P13.curiosidad, v053.P13.incitación_de_los_medios_de_comunicación, } \\
\text { v053.P13.ninguna, v053.P13.presión_de_la_pareja, v053.P13.presión de las } \\
\text { amistades }\end{array}$ \\
\hline $\begin{array}{l}\text { ¿Qué método } \\
\text { anticonceptivo usas? }\end{array}$ & $\begin{array}{l}\text { v059.P19.dispositivos, v059.P19.inyecciones, v059.P19.pastillas, } \\
\text { v059.P19.preservativo, v059.P19.ninguna, v059.P19.otros }\end{array}$ \\
\hline $\begin{array}{lr}\text { ¿Cuál } & \text { método } \\
\text { anticonceptivo } & \text { no } \\
\text { utilizarías? } & \end{array}$ & $\begin{array}{l}\text { v062.P22.anticonceptivo de emergencia, v062.P22.anticonceptivo oral, } \\
\text { v062.P22.coito interrumpido, v062.P22.DUI, v062.P22.inyecciones, } \\
\text { v062.P22.preservativos, v062.P22.ritmo menstrual, v062.P22.ninguno }\end{array}$ \\
\hline $\begin{array}{l}\text { ¿Le ha diagnosticado el } \\
\text { médico alguna } \\
\text { enfermedad de } \\
\text { transmisión sexual? }\end{array}$ & $\begin{array}{l}\text { v066.P26.gonorrea, v066.P26.ladillas, v066.P26.micosis, } \\
\text { v066.P26.tricomonas, v066.P26.ninguna }\end{array}$ \\
\hline $\begin{array}{l}\text { ¿Hace cuánto tiempo } \\
\text { tuvo esta enfermedad? }\end{array}$ & $\begin{array}{l}\text { v067.P27.en_los_ultimos_12_meses, v067.P27.hace_5_anos_o_mas, } \\
\text { v067.P27.hace_mas_de_1_ano_pero_menos_5, v067.P27.ninguno }\end{array}$ \\
\hline $\begin{array}{l}\text { ¿Cuándo tuvo su } \\
\text { primera relación sexual } \\
\text { cree que su pareja tenía } \\
\text { relaciones con alguien } \\
\text { más? }\end{array}$ & $\begin{array}{l}\text { v079.P39.no_sabia, v079.P39.no_yo_lo_sabia, v079.P39.probablemente } \\
\text { pero_yo_no_lo_sabia, v079.P39.probablemente si pero_yo_no_lo_sabia, } \\
\text { v079.P39.si_yo_lo_sabia, v079.P39.ninguna }\end{array}$ \\
\hline
\end{tabular}

La figura 3 muestra el árbol de similaridad, en él se puede apreciar la formación de dos grandes grupos o clases que engloban algunas variables y que establecen las conexiones de similaridad entre las diferentes clases de variables. El grupo de la izquierda está conformado por la variable presión de las amistades asociado a tener su primera relación sexual y el uso de dispositivos como método anticonceptivo que se asocia en un séptimo nivel con cuando tuvieron su primera relación sexual creen que probablemente su pareja tenía relaciones con alguien más pero que no lo sabían. En el grupo de la izquierda se relaciona en sexto nivel, conformado por pasión como la primera palabra que asocian con sexo y que no sabía si su pareja tenía relaciones con alguien más cuando tuvo su primera relación sexual, asociada en un octavo nivel con amor observándose una baja similaridad. En esta clase también se relaciona la influencia de las amistades como un motivo para tener por primera vez relaciones sexuales, usan dispositivos como método anticonceptivo y que existe la probabilidad que su pareja tenía relaciones con alguien más. La segunda conexión se establece entre pasión, desconocimiento de que su pareja podía tener relaciones con alguien más en su primera relación sexual y baja similaridad con el amor. El grupo de la derecha está compuesto por 
dos subclases, la primera con un nodo significativo en un primer nivel conformado por tricomoniasis como Infección de Transmisión Sexual (ITS) diagnosticada por un médico y que probablemente su pareja tenía relaciones con alguien más en su primera relación sexual pero que no lo sabían, agrupando a las anteriores en un tercer nivel la incitación de los medios de comunicación que le motivaron e impulsaron a tener la primera relación sexual. A todas las conexiones descritas se asocia en un cuarto nivel de similaridad a que sí probablemente su pareja tenía relaciones con alguien más en su primera relación pero que no lo sabía. Este grupo asocia también el placer como la primera palabra que relacionan con el sexo y que no cree que su pareja tenía relaciones con alguien más cuando tuvo su primera relación sexual ya que lo sabía. Además, se unen inicialmente una ITS la tricomoniasis, la duda que su pareja tenía relaciones con alguien más en su primera relación sexual, el motivo para tener su primera relación sexual la incitación de los medios de comunicación, todas éstas con probablemente su pareja si tenía relaciones con alguien más en su primera relación pero que no lo sabía y la segunda conexión se establece entre placer y que no cree que su pareja tenga relaciones con alguien más en su primera relación sexual. El índice de conexión entre las dos clases principales es bajo lo que muestra que existe una moderada similaridad.

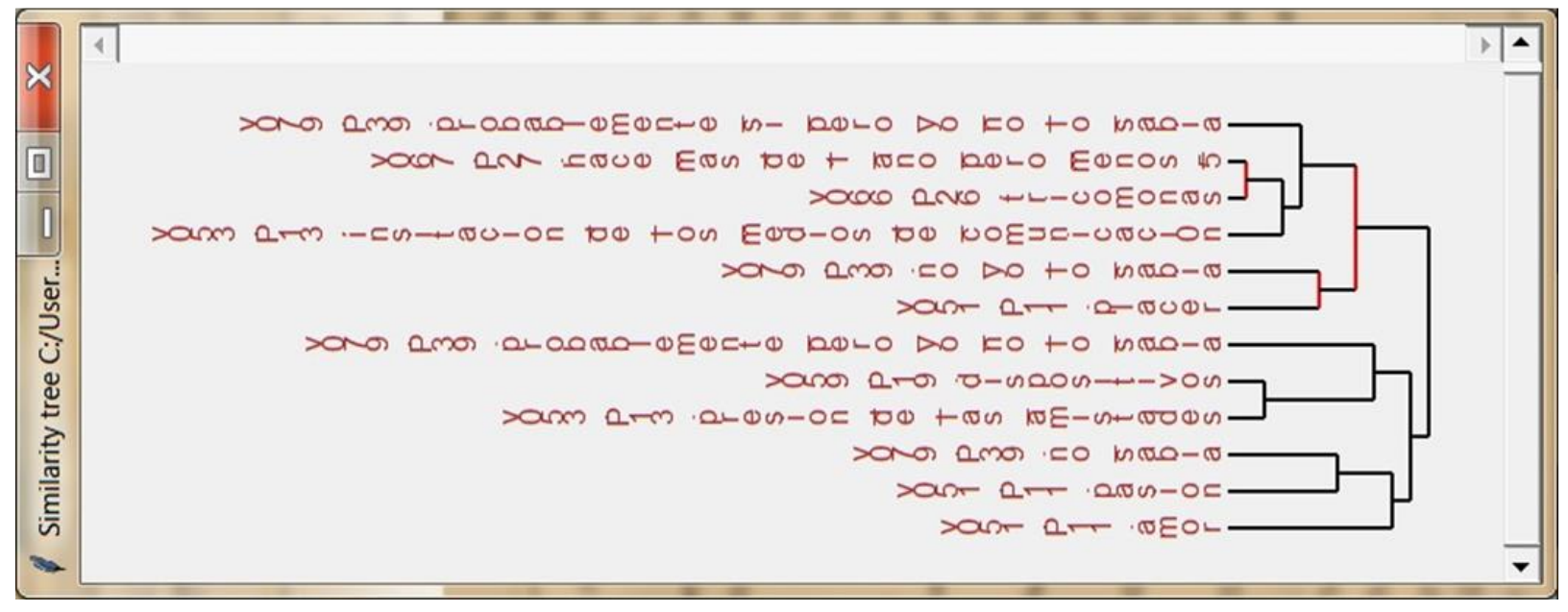

Figura 3. Arbol de Similaridad generado por R-CHIC, para los datos de percepción sexual

La tabla 2, muestra algunas de las reglas de clasificación generadas automáticamente por el software estadístico R-CHIC. Además, su nivel de cohesión y su interpretación en el contexto de la percepción sexual universitaria.

Tabla 2. Algunas preguntas y sus posibles respuestas de la entrevista no estructurada sobre percepción sexual. 


\section{OCincia Digital}

ISSN: 2602-8085

Vol. 2, N4.1, p. 122-139, octubre - diciembre, 2018

\begin{tabular}{|c|c|c|c|}
\hline No & Clasificación & Interpretación & Coh \\
\hline 1 & (v051.P11.amor v051.P11.pasión) & $\begin{array}{l}\text { Significa que la primera palabra que asocian con sexo } \\
\text { es amor y relaciona con pasión. }\end{array}$ & 1 \\
\hline 2 & $\begin{array}{l}((\mathrm{v} 051 . P 11 . a m o r \\
\text { v051.P11.pasión) } \\
\text { v051.P11.placer })\end{array}$ & $\begin{array}{l}\text { Significa que sexo asocian con palabras amor y pasión } \\
\text { asocia también con placer. }\end{array}$ & 1 \\
\hline 3 & $\begin{array}{l}\text { (((v051.P11.amor } \\
\text { v051.P11.pasión) } \\
\text { v051.P11.placer) } \\
\text { v053.P13.incitación de los medios } \\
\text { de comunicación) }\end{array}$ & $\begin{array}{l}\text { Significa que sexo asocian con amor, pasión, placer } \\
\text { que relaciona con la incitación de los medios de } \\
\text { comunicación como un motivo para tener su primera } \\
\text { relación sexual. }\end{array}$ & 1 \\
\hline 4 & $\begin{array}{l}\text { ((((v051.P11.amor } \\
\text { v051.P11.pasión) } \\
\text { v051.P11.placer) } \\
\text { v053.P13.incitación de los medio } \\
\text { de comunicación) } \\
\text { v053.P13.presión de las } \\
\text { amistades) }\end{array}$ & $\begin{array}{l}\text { Significa que sexo asocian con palabras como amor, } \\
\text { pasión, placer, persuadido por los medios de } \\
\text { comunicación asocian también con la presión que } \\
\text { ejercen las amistades como un motivo para tener su } \\
\text { primera relación sexual. }\end{array}$ & 1 \\
\hline 5 & $\begin{array}{l}\text { ((((v051.P11.amor } \\
\text { v051.P11.pasión) } \\
\text { v051.P11.placer) v053.P13. } \\
\text { incitación de los medios de } \\
\text { comunicación) v053.P13. presión } \\
\text { de las amistades) } \\
\text { v059.P19.dispositivos) }\end{array}$ & $\begin{array}{l}\text { Significa que sexo asocian con amor, pasión, placer, } \\
\text { que está influenciado por los medios de comunicación } \\
\text { y las amistades para tener su primera relación sexual, } \\
\text { asocian con el uso de dispositivos como método } \\
\text { anticonceptivo. }\end{array}$ & 1 \\
\hline 6 & $\begin{array}{l}(((((\mathrm{v} 051 . P 11 . a m o r \\
\text { v051.P11.pasión) } \\
\text { v051.P11.placer) v053.P13. } \\
\text { incitación de los medios de } \\
\text { comunicación) v053.P13. presión } \\
\text { de las amistades) } \\
\text { v059.P19.dispositivos) } \\
\text { v059.P19.ninguna) }\end{array}$ & $\begin{array}{l}\text { Significa que sexo asocian con amor, pasión, placer, } \\
\text { que está influenciado por los medios de comunicación } \\
\text { y las amistades para tener su primera relación sexual } \\
\text { con el uso de dispositivos como método } \\
\text { anticonceptivo, asocian también con ninguno. }\end{array}$ & 1 \\
\hline 7 & $\begin{array}{l}\text { ((((((v051.P11.amor } \\
\text { v051.P11.pasión) } \\
\text { v051.P11.placer) v053.P13. } \\
\text { incitación de los medios de } \\
\text { comunicación) v053.P13. presión } \\
\text { de las amistades }) \\
\text { v059.P19.dispositivos) } \\
\text { v059.P19.ninguna) } \\
\text { v059.P19.otros) }\end{array}$ & $\begin{array}{l}\text { Significa que sexo asocian con amor, pasión, placer, } \\
\text { que está influenciado por los medios de comunicación } \\
\text { y las amistades para tener su primera relación sexual } \\
\text { con el uso de dispositivos como método } \\
\text { anticonceptivo, existen estudiantes que no utilizan } \\
\text { ningún método anticonceptivo, asocian también con } \\
\text { otros. }\end{array}$ & 1 \\
\hline 11 & $\begin{array}{l}\text { (v051.P11.caricias } \\
\text { v059.P19.inyecciones) }\end{array}$ & $\begin{array}{l}\text { Significa que caricias es la primera palabra que asocian } \\
\text { con sexo, relacionan con las inyecciones como el } \\
\text { método anticonceptivo que usan. }\end{array}$ & 1 \\
\hline 12 & $\begin{array}{l}((\mathrm{v} 051 . P 11 . \text { caricias } \\
\text { v059.P19.inyecciones }) \\
\text { v062.P22.anticonceptivo oral })\end{array}$ & $\begin{array}{l}\text { Significa que caricias es la primera palabra que asocian } \\
\text { con sexo, usan inyecciones como método }\end{array}$ & 1 \\
\hline
\end{tabular}




\begin{tabular}{|c|c|c|c|}
\hline & & $\begin{array}{l}\text { anticonceptivo, relacionan con anticonceptivo oral } \\
\text { como método anticonceptivo que usan. }\end{array}$ & \\
\hline 13 & $\begin{array}{l}\text { (v051.P11.cariño } \\
\text { v059.P19.pastillas) }\end{array}$ & $\begin{array}{l}\text { Significa que cariño es la primera palabra que asocian } \\
\text { con sexo, relacionan con pastillas como el método } \\
\text { anticonceptivo que usan }\end{array}$ & 1 \\
\hline 14 & $\begin{array}{l}\text { (v051.P11.comprensión } \\
\text { v062.P22.coito interrumpido) }\end{array}$ & $\begin{array}{l}\text { Significa que la comprensión es la primera palabra que } \\
\text { asocian con sexo, relacionan con coito interrumpido } \\
\text { como el método anticonceptivo que no utilizarían }\end{array}$ & 1 \\
\hline 15 & $\begin{array}{l}\text { (v051.P11.deseo } \\
\text { v062.P22.anticonceptivo de } \\
\text { emergencia) }\end{array}$ & $\begin{array}{l}\text { Significa que deseo es la primera palabra que asocian } \\
\text { con sexo, relacionan con anticonceptivo de emergencia } \\
\text { como el método anticonceptivo que usan }\end{array}$ & 1 \\
\hline 16 & $\begin{array}{l}\text { (v051.P11.felicidad } \\
\text { v062.P22.ritmo menstrual) }\end{array}$ & $\begin{array}{l}\text { Significa que la felicidad es la primera palabra que } \\
\text { asocian con sexo, relacionan con ritmo menstrual como } \\
\text { el método anticonceptivo que no utilizarían. }\end{array}$ & 1 \\
\hline 17 & $\begin{array}{l}\text { (v051.P11.hombre v079.P39.no } \\
\text { sabia) }\end{array}$ & $\begin{array}{l}\text { Significa que hombre es la primera palabra que asocian } \\
\text { con sexo, relacionan con no sabían que su pareja tenía } \\
\text { relaciones con alguien más cuando tuvo su primera } \\
\text { relación sexual. }\end{array}$ & 1 \\
\hline 18 & $\begin{array}{l}\text { (v051.P11.mujer } \\
\text { v062.P22.inyecciones) }\end{array}$ & $\begin{array}{l}\text { Significa que mujer es la primera palabra que asocia } \\
\text { con sexo, relacionan con las inyecciones como el } \\
\text { método anticonceptivo que usan }\end{array}$ & 1 \\
\hline 19 & $\begin{array}{l}\text { (v051.P11.vida v079.P39.no yo lo } \\
\text { sabía) }\end{array}$ & $\begin{array}{l}\text { Significa que vida es la primera palabra que asocian } \\
\text { con sexo, relacionan con no cree que su pareja tenía } \\
\text { relaciones con alguien más cuando tuvo su primera } \\
\text { relación sexual, lo sabían. }\end{array}$ & 1 \\
\hline 20 & $\begin{array}{l}\text { v067.P27.ninguno } \\
\text { v051.P11.satisfacción) }\end{array}$ & $\begin{array}{l}\text { Significa que en ningún momento tuvieron Infección } \\
\text { de Transmisión Sexual (ITS), asocian con satisfacción } \\
\text { que es la primera palabra que relacionan con sexo. }\end{array}$ & 1 \\
\hline 21 & $\begin{array}{l}\text { (v066.P26.ninguna } \\
\text { v053.P13.ninguna) }\end{array}$ & $\begin{array}{l}\text { Significa que no le han diagnosticado el médico } \\
\text { ninguna infección de transmisión sexual, asocia con } \\
\text { ninguno de los siguientes motivos curiosidad, } \\
\text { incitación de los medios de comunicación, presión de } \\
\text { la pareja o de las amistades le impulsaron a tener tu } \\
\text { primera relación sexual. }\end{array}$ & 1 \\
\hline 26 & $\begin{array}{l}\text { ((v051.P11.mujer } \\
\text { v062.P22.inyecciones) } \\
\text { (v051.P11.deseo } \\
\text { v062.P22.anticonceptivo_de_eme } \\
\text { rgencia)) }\end{array}$ & $\begin{array}{l}\text { Significa que mujer es la primera palabra que asocia } \\
\text { con sexo, las inyecciones es el método anticonceptivo } \\
\text { que usan, relacionan con el deseo como la primera } \\
\text { palabra que asocian con sexo, el anticonceptivo de } \\
\text { emergencia es el método anticonceptivo que usan. }\end{array}$ & 0.999998 \\
\hline 27 & $\begin{array}{l}\text { (v066.P26.gonorrea } \\
\text { ((v051.P11.mujer } \\
\text { v062.P22.inyecciones) } \\
\text { (v051.P11.deseo } \\
\text { v062.P22.anticonceptivo_de_eme } \\
\text { rgencia))) }\end{array}$ & $\begin{array}{l}\text { Significa que le han diagnosticado el médico gonorrea } \\
\text { como infección de transmisión sexual, relacionan con } \\
\text { mujer como la primera palabra asociada con sexo y las } \\
\text { inyecciones como el método anticonceptivo que usan, } \\
\text { paralelamente la palabra deseo con sexo y el uso del } \\
\text { anticonceptivo de emergencia }\end{array}$ & 0.999994 \\
\hline
\end{tabular}

La Figura 4, muestra en la representación gráfica (dendograma) algunas de las reglas de clasificación generadas automáticamente por el software estadístico R-CHIC. 
Vol. 2, N4.1, p. 122-139, octubre - diciembre, 2018

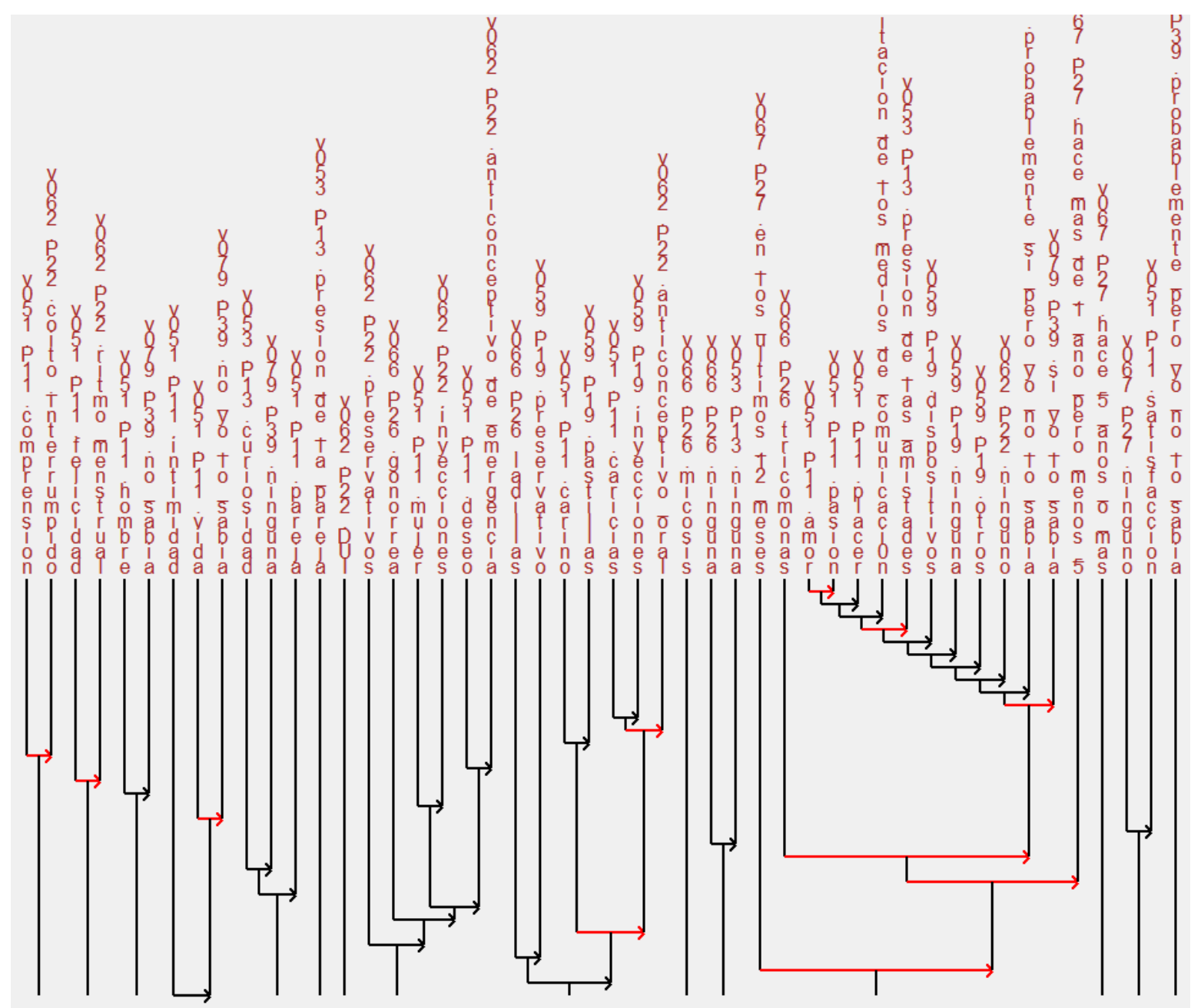

Figura 4. Árbol de jerárquico generado por R-CHIC, para los datos de percepción sexual

A continuación se muestran e interpretan las R-reglas obtenidas automáticamente con el software libre R-CHIC y que se puede observar en la Figura 5.

Regla R1: Los estudiantes que relacionan la palabra amor, pasión, placer con sexo no sabían que cuando tuvieron su primera relación sexual que su pareja tenía relaciones con alguien más, con una intensidad implicativa comprendida entre $60 \%$ y $65 \%$,

Regla R2: A los estudiantes que les diagnosticó el médico tricomoniasis como infección de transmisión sexual fue hace más de un año pero menos de 5 con una intensidad implicativa del $80 \%$, los cuales relacionaron el sexo con el placer con una intensidad implicativa del $65 \%$, y no creen que su pareja en su primera relación sexual tenía relaciones con alguien más porque lo sabían, con una intensidad del $65 \%$.

Regla R3: A los estudiantes que les diagnosticó el médico tricomoniasis como infección de transmisión sexual fue hace más de un año pero menos de 5 con una intensidad implicativa del $80 \%$, fueron impulsados a tener su primera relación sexual por la incitación de los medios de comunicación con una intensidad implicativa del $65 \%$ y si creen que en su primera 
relación sexual su pareja tenía relaciones con alguien más, pero que no lo sabían con una intensidad implicativa del 65\%,

Regla R4: Los estudiantes que no utilizarían el método anticonceptivo de dispositivos, fueron impulsados a tener su primera relación sexual por presión de las amistades con una intensidad implicativa del $80 \%$.

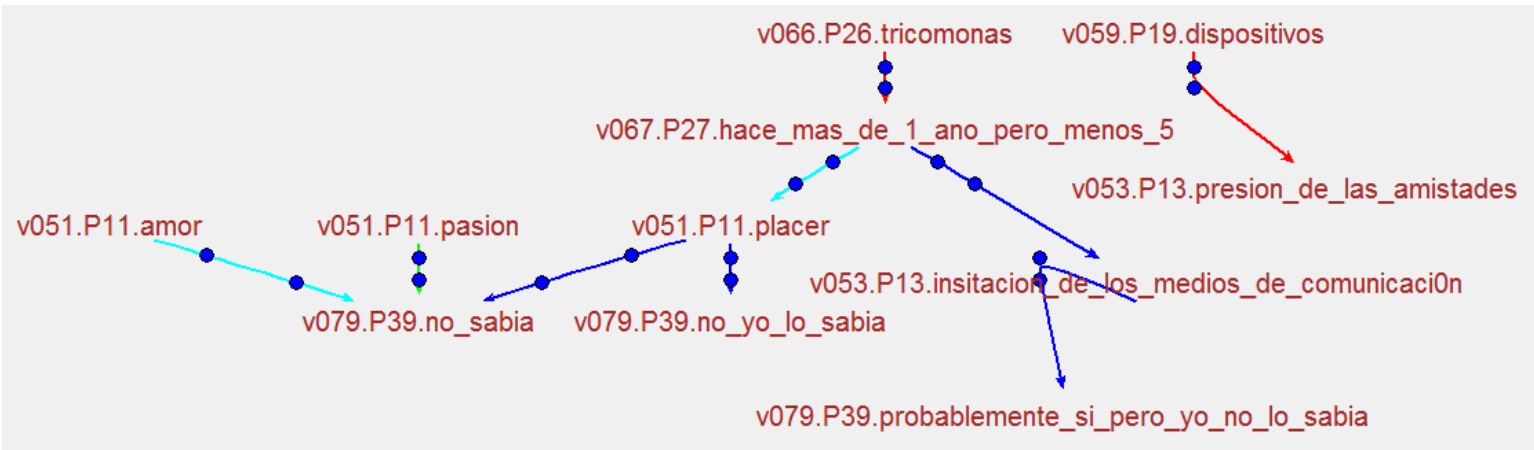

Figura 5. Grafo implicativo generado por R-CHIC, para los datos de percepción sexual

\section{Conclusiones.}

En este trabajo se llevó a cabo un análisis implicativo y de similaridades de los resultados obtenidos en una encuesta sobre percepción sexual aplicado a estudiantes universitarios. Las relaciones implicativas ponen de manifiesto la implicación de unas pruebas sobre otras, y las de similaridad indican la homogeneidad entre variables. Los resultados de los gráficos de similaridad evidencian dos grandes grupos de similaridad. Uno que incluye el motivo que les impulsaron para tener por primera vez relaciones sexuales fue la influencia de las amistades, uso de dispositivos como método anticonceptivo, la probabilidad que su pareja tenía relaciones con alguien más, la pasión, el amor y el desconocimiento de que su pareja tenía relaciones con alguien más en su primera relación sexual. Otro que incluye la tricomoniasis como una Infección de Transmisión Sexual (ITS) diagnosticada por un médico, la duda que su pareja tenía relaciones con alguien más en su primera relación sexual, un motivo que le impulsó para tener su primera relación sexual fue la incitación de los medios de comunicación, la probabilidad de que su pareja si tenía relaciones con alguien más en su primera relación pero que no lo sabía, el placer como la primera palabra que asocia con sexo, que no cree que su pareja tenía relaciones con alguien más en su primera relación sexual. Los gráficos muestran poca similaridad en la conexión entre estos dos grupos. El análisis implicativo muestra que se forman dos grupos de implicación cuando se estudian los resultados. El nivel de significación máximo es de $80 \%$. Mayor grado de influencia tiene la ITS tricomoniasis al iniciar el árbol implicativo, pero no existe implicaciones con el método anticonceptivo de uso de dispositivos el mismo que solo influye en la presión de las amistades como el motivo que le impulsaron a tener su primera relación sexual.

Se han realizado interpretaciones importantes en la percepción sexual estudiantil basándonos 
en el Análisis Estadístico Implicativo, mediante reglas de relación simétricas y asimétricas. Además, el software R-CHIC permite en forma rápida, visual, intuitiva y comprensible el descubrimiento de conocimiento en la percepción sexual estudiantil.

\section{Agradecimientos.}

Los autores agradecen a la Escuela Superior Politécnica de Chimborazo que mediante el apoyo al proyecto "Análisis e innovación de las investigaciones en la Facultad de Ciencias como estrategia para incrementar la producción científica", permite que se analice, mejore e incremente la investigación en la Facultad de Ciencias y en la ESPOCH, a los investigadores del grupo de investigación en Ciencia de Datos - CIDED, a Shirley Armas Analuisa por su ayuda en la binarización de los datos.

\section{Referencias bibliográficas.}

Adela Montero, M. V. (2011). Educación sexual: Un pilar fundamental en la sexualidad de la adolescencia. Revista Medica de Chile.

Bioconductor - Rgraphviz.

Cabrera, A. R., Ramos, G. S., Palú, M. E. C., \& Cáceres, B. P. (2013). Estrategia educativa sobre promoción en salud sexual y reproductiva para adolescentes y jóvenes universitarios. Revista Cubana de Salud Publica.

Calero, J. L., \& Santana, F. (2001). Percepciones de un grupo de adolescentes sobre iniciaci??n sexual, embarazo y aborto. Revista Cubana de Salud Publica.

Ciencias, U. D. E., Granma, M., Universitario, P., Hirzel, J., \& Granma, B. (2016). Conocimientos sobre sexualidad en adolescentes estudiantes de medicina . Bayamo , 2014 Knowledg e on sexuality by adolescent's medical students . Bayamo. 20(5), 5269.

COUTURIER, R. (2000). TRAITEMENT DE L'ANALYSE STATISTIQUE DANS CHIC. ASI1, 9.

Couturier, R. (2007). CHIC: utilización y funcionalidades. ASI4, 14.

Couturier, R., \& Almouloud, S. A. (2009). Historique et fonctionnalités de CHIC. 
Couturier, R., \& Pazmiño, R. (2016). Use of Statistical Implicative Analysis in Complement of Item Analysis. International Journal of Information and Education Technology, 6(1), 39.

Dávila León, O. (2004). ADOLESCENCIA Y JUVENTUD: DE LAS NOCIONES A LOS ABORDAJES. Ultima década. doi: 10.4067/s0718-22362004000200004

Delgado, J. R. P., Flores, M. I. B., Palos, P. A., Adolescentes, \& Consumo De Alcohol, Y. C. S. D. R. E. (2007). CONSUMO DE ALCOHOL Y CONDUCTA SEXUAL DE RIESGO EN ADOLESCENTES (Vol. 18).

Folch, C., Álvarez, J. L., Casabona, J., Brotons, M., \& Castellsagué, X. (2015). Determinantes de las conductas sexuales de riesgo en jovenes de cataluña. Revista Espanola de Salud Publica. doi: 10.4321/s1135-57272015000500005

Gras, R. (2014). Genèse et développement de l'Analyse Statistique Implicative ; rétrospective historique. Educação Matemática Pesquisa, 16(3), 645-661.

GRAS, R., \& COUTURIER, R. (2012). Entropic implication and causality. ASI6, 12.

Guerra Electra, M. M. V. I. S. M. C. S. (2016). Conocimientos sobre sexualidad en adolescentes estudiantes de medicina. Multimed. Revista Médica. Granma, 20(5), 5269.

Hernández, S., del Carmen, R., Pérez Piñero, J., \& Sanabria Ramos, G. (2013). Vivencias de la sexualidad en estudiantes universitarios. Revista Cubana de Salud Pública, 39, 915928.

Hurtado, T., Zabalgoitia, M., \& Olvera, J. (2013). Conocimientos Y Actitudes Sobre. Artículo, 16(1), 258-268.

Inicio - Escuela Superior Politécnica de Chimborazo.

Pazmiño-Maji, R. A., García-Peñalvo, F. J., \& Conde-González, M. A. (2016). Approximation of statistical implicative analysis to learning analytics: a systematic review. Paper presented at the Proceedings of the Fourth International Conference on Technological Ecosystems for Enhancing Multiculturality.

Pazmiño-Maji, R. A., García-Peñalvo, F. J., \& Conde-González, M. A. (2017). Statistical Implicative Analysis aproximation to KDD and Data Mining: A systematic and mapping review in Knowledge Discovery Database framework. 
Vol. 2, N4.1, p. 122-139, octubre - diciembre, 2018

Pazmiño, R. (2014). Aproximación al Análisis Estadístico Implicativo desde sus Aplicaciones Educativas.

Rchic - | Raphael Couturier.

Regnier, J.-C. (2009). Test de Mac Nemar et Analyse statistique implicative. In G. Régis, R. Jean-Claude \& G. Fabrice (Eds.), Analyse statistique implicative: Une méthode d'analyse de données pour la recherche de causalités. (pp. 271-277): Éditions Cépaduès.

Régnier, J.-C., \& Gras, R. (2015). Extension of the implicative statistical analysis to any continuous variable. In R. Jean-Claude (Ed.), analyse statistique implicative: des sciences dures aux sciences humaines et sociales (pp. 130-142). Radès Tunisie: ARSA Association pour la Recherche en Statistique Appliquée.

Ribeiro, R. A., Santos, M. M. d., Vilardell, N. P., Carolei, O., \& Mengali, N. M. (2015). Uso do CHIC na Análise de Praticasen Educacao e Tecnologia. Uso do CHIC na Formação de Educadores. 
Para citar el artículo indexado.

Pazmiño R., Bonilla M., Baquero J., \& Miguez R.. (2018). Software estadístico chic: descubriendo sus potencialidades mediante el análisis de percepción sexual universitaria. Revista electrónica Ciencia Digital 2(4.1), 122-139. Recuperado desde: http://cienciadigital.org/revistacienciadigital2/index.php/CienciaDigital/article/view/195

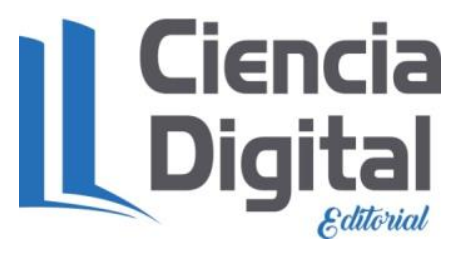

El artículo que se publica es de exclusiva responsabilidad de los autores y no necesariamente reflejan el pensamiento de la Revista Ciencia Digital.

El articulo queda en propiedad de la revista y, por tanto, su publicación parcial y/o total en otro medio tiene que ser autorizado por el director de la Revista Ciencia Digital.
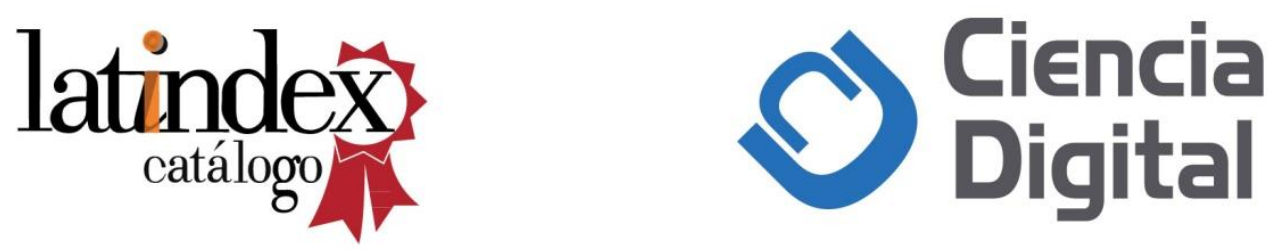